\title{
Caracterização das forças de impacto na execução do Jump Kick
}

\author{
Guilherme Felício Mülbersted Coelho ${ }^{1}$ \\ Saray Giovana dos Santos ${ }^{1,2}$ \\ ${ }^{1}$ Departamento de Educação Física, Centro de Desportos da UFSC, Florianópolis, SC, \\ Brasil \\ ${ }^{2}$ Programa de Pós-Graduação em Educação Física da UFSC, Florianópolis, SC, Brasil
}

\begin{abstract}
Resumo: Este estudo objetivou identificar o número de Jump Kick realizados pelos professores por aula (Mix 30), as magnitudes e tempos dos impactos no tornozelo e joelho na aterrissagem; comparar os impactos e relacioná-los com massa corporal, estatura e tempo de prática. Participaram oito professores com 28,12 $\pm 6,46$ anos, $171,0 \pm 6,12 \mathrm{~m}, 68,38 \pm 9,04 \mathrm{~kg}$ e com $41 \pm 32,23$ meses de experiência no Body Combat. Foi utilizada uma entrevista semi-estruturada, filmadora, balança digital, antropômetro e um acelerômetro triaxial. Os professores realizaram 1420 saltos por aula; as maiores magnitudes de impacto ocorreram no tornozelo (31,4g, 27,6 g e 21,6 g nos eixos $x, y$ e $z$ respectivamente); o maior tempo de impacto ocorreu no joelho $(0,028 \mathrm{~s}$ eixo $x)$; encontraram-se diferenças significativas nos valores de impactos entre os professores; não foram encontradas correlações significativas $(p \leq 0,05)$ entre as variáveis. Mesmo sendo os impactos na aterrissagem do Jump Kick de baixa magnitude e curta duração, a repetitividade de saltos apresentada é um fator que poderá gerar futuras lesões.
\end{abstract}

Palavras-chave: Prevenção e controle. Cinética. Ginástica.

\section{Characterization of impact forces for implementing of Jump Kick}

\begin{abstract}
This study aimed to identify the number of jumps in Jump Kick training (Mix 30), the magnitudes and duration time of the impacts generated in the knee and ankle during landing; compare the magnitudes and associate them with weight, height and practice time. Eight teachers with $28,12 \pm 6,46$ years; height $171,0 \pm 6,12 \mathrm{~m}$; weight $68,38 \pm 9,04 \mathrm{~kg}$ and $41 \pm 32,23$ months of practice time in Body Combat participated in this study. Was used a semi-structured interview, a video camera, a digital balance and a tri-axial accelerometer. The teachers realized 1420 jumps per class; the biggest impact magnitudes occurred in the ankle $(31,4 g, 27,6 \mathrm{~g}$ e $21,6 \mathrm{~g}$ in axis $x, y$ e $z$ respectively); the biggest impact time occurred in the knee $(0,028 \mathrm{~s}, x$ axis); there was difference in impact values between teachers; there was any correlation $(p \leq 0,05)$ between variables. Even if the impact values are low, the high impact numbers during a day is a factor that can cause future injuries.
\end{abstract}

Key Words: Prevention and control. Kinetics. Gymnastics.

\section{Introdução}

A busca pelas respostas do corpo humano aos impactos e vibrações, principalmente nos membros inferiores, que são advindas das aterrissagens em diferentes tipos de saltos, tem sido tema de pesquisa em diferentes modalidades esportivas (HAMILL et al., 1995; DUFEK; ZHANG, 1996; LAFORTUNE et al., 1996; NIGG, 1997; MAHAR et al., 1997; DERRICK et al., 1998; MIZRAHI et al., 2000; NIGG; WAKELING, 2001; PANDA, 2001; MERCER et al., 2003; COVENTRY et al., 2006;

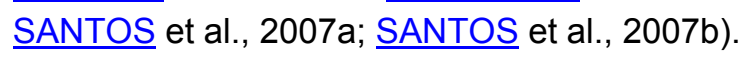

Um atleta, dependendo da atividade desportiva que realiza, pode produzir níveis de aumento de força vertical que podem chegar de 12 vezes o seu peso corporal (PC) ( $\underline{\text { NIGG; }}$ WAKELING, 2001) até 16 PC (AMADIO, 1999), como ocorre na segunda fase do salto triplo do atletismo, e até 14,4 PC na ginástica olímpica, correspondendo a valores de aceleração de impacto de até $200 \mathrm{~g}$. Fantini e Menzel (2001) citam que com as técnicas de aterrissagem mais apuradas de saltos no voleibol, os impactos máximos não ultrapassam 4,5 PC, no entanto, com técnicas não apuradas, este chegam entre 6 e 7 PC. Já no basquetebol, os valores de impacto na aterrissagem chegam a 5,56 PC ( $\underline{\text { SACCO }}$ et al., 2004), e no step training, até 2,04 PC (PANDA, 2001).

Poucos estudos investigaram os impactos gerados nos membros inferiores durante aterrissagens em atividades esportivas. $O$ impacto mecânico do atleta contra o solo, como ocorre durante a aterrissagem de saltos, é uma vibração não periódica na forma de pulso e é chamado de evento transitório que acontece numa fração de segundos (milissegundos) 
(SANTOS, 2003). As relações entre as magnitudes de impactos durante aterrissagens e as lesões dos atletas foram investigadas nos

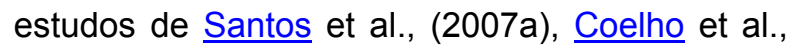
(2007), Santos et al., (2007b), Piucco e Santos (2009), demonstrando que a técnica utilizada para o amortecimento do impacto é de suma importância para a dissipação de energia. Embora nos estudos citados algumas magnitudes de impacto encontradas não estejam em níveis causadores de lesões, quando analisadas pelo critério de Macaulay (1987), é a repetição dos eventos impactantes por um longo período de treinamento que pode gerar danos aos componentes biomateriais (DERRICK et al. 1998).

Por outro lado, os impactos não ocorrem apenas nos esportes, mas também em atividades físicas praticadas em academias, como os diferentes tipos de ginásticas da franquia Body Systems. De acordo com Brauner (2007), o sistema de franquia denominada Body Systems é uma forma de ginástica inovadora que revolucionou o mercado desta prática, ampliandoo muito rapidamente.

Um dos tipos de prática do Body Systems é o Body Combat, que é um sistema ginástico que utiliza movimentos de artes marciais, combinados com músicas e diferentes patamares de treinamento. Este sistema tem como objetivo trabalhar o condicionamento físico através de um altíssimo gasto calórico, proporcionando definição muscular, trabalhando a defesa pessoal $e$ apresentando um alto grau de motivação.

Uma aula de Body Combat é dividida em três fases que obedecem a uma seqüência lógica, sendo elas: a) fase de aquecimento para membros superiores e inferiores a partir de exercícios aeróbios de baixo impacto, com enfoque no aumento da temperatura corporal e da mobilidade, para que assim os participantes possam se adaptar às técnicas básicas do Body Combat, acostumando-se lentamente e seguindo o instrutor sem maiores problemas; b) fase intermediária, composta por seis músicas, sendo que três delas trabalham o treinamento de alta intensidade, com sobrecargas de trabalho aeróbio e anaeróbio, e as outras três trabalham exercícios de combate com intensidade de média a alta. É nesta fase que a técnica Jump Kick, objeto de estudo desta pesquisa, é executada no Mix 30 (aula com coreografia pré-estipulada); c) fase final, em que exercícios abdominais complementam o treinamento e conduzem os participantes ao esfriamento, permitindo que $o$ sistema cardiovascular ajuste o fluxo sangüíneo, neutralize o ácido lático e restabeleça o equilíbrio químico corporal (MILLS, 2002).

Vale ressaltar que os Mix são trocados a cada três meses, e antes de executar essa sessão, os professores são treinados por instrutores $\mathrm{e}$ coreógrafos profissionais, juntamente com um material didático, no qual podem observar os detalhes de cada movimento descrito. Tendo em vista que o método Body Combat é baseado em modalidades de autodefesa tais como o karatê, o taekwondo, o tai chi chuan e o muay thai, ele exige dos seus praticantes movimentos de mudança de direção rápida, como saltos, socos e chutes. Estes movimentos geram impactos de maneira repetitiva nos membros inferiores dos praticantes durante as aterrissagens, e com magnitudes desconhecidas até então.

Deste modo, baseando-se nos pressupostos teóricos que apontam os movimentos impactantes e repetitivos como causadores de lesões, e preocupado com a integridade física dos profissionais que ministram Body Combat, este estudo buscou investigar as características de prática, inerentes aos movimentos impactantes e repetitivos, dos profissionais que ministram Body Combat. Dessa forma objetivouse identificar o número de saltos realizados pelos professores no Mix 30; identificar as magnitudes e os tempos dos impactos recebidos pelos membros inferiores (tornozelo e joelho) dos professores na fase de aterrissagem do salto Jump Kick; comparar as magnitudes dos impactos entre os sujeitos, entre os eixos e entre as articulações; e relacionar as magnitudes dos impactos com a massa corporal, com a estatura e com o tempo de prática dos mesmos.

\section{Materiais e Métodos}

Participaram deste estudo oito professores de Educação Física, sendo cinco homens e três mulheres, com média de idade de 28,12 $\pm 6,46$ anos; estatura de $171,0 \pm 6,12 \mathrm{~m}$; massa corporal $68,38 \pm 9,04 \mathrm{~kg}$ e com experiência na prática do Body Combat de 41 $\pm 32,23$ meses.

Como instrumentos de medidas foram utilizados: uma entrevista semi-estruturada com a finalidade de identificar as características dos professores; uma filmadora da marca Sony ${ }^{\circledR}$ com 
capacidade de 30 quadros por segundo, para identificar o número, a freqüência, o intervalo de repetições do movimento Jump Kick e também o número de saltos realizados em uma sessão de Body Combat; uma balança digital de marca Toledo ${ }^{\circledR}$ modelo 2096 PP com sensibilidade de 50 gramas para medir a massa corporal; um antropômetro de marca CESCORF, com 2000 $\mathrm{mm}$ e graduação milimétrica, para as medidas de estatura; um acelerômetro triaxial do tipo 4321, da Brüel \& Kjaer $^{\mathrm{TM}}$ confeccionado de titânio, com dimensões de $28,6 \times 28,6 \times 17 \mathrm{~mm}$, com capacidade máxima de choque de $10000 \mathrm{~m} / \mathrm{s}^{2}$ ou $1000 \mathrm{~g}$ massa de $56,1 \mathrm{~g}$ e freqüência natural de $40 \mathrm{kHz}$, para medir os impactos e vibrações resultantes das colisões dos membros inferiores (joelhos e tornozelos) dos professores com o piso; três pré-amplificadores da Brüel \& $\mathrm{Kjaer}^{\mathrm{TM}}$ modelo 2635 para os eixos $x$ (antero-posterior) e $y$ (latero-lateral) e modelo 2626 para o eixo $z$ (vertical), um módulo condicionador de sinais MCS1000 Lynxs ${ }^{\circledR}$, composto por 16 canais de até $+10 \mathrm{~V}$ seguido de um conversor analógico digital $(A D)$, para aquisição dos sinais via acelerometria.

Antes das coletas, os professores assinaram o termo de consentimento livre e esclarecido, conforme prevê as Resoluções 196 e 251, de 07/08/97 do Conselho Nacional da Saúde (análise do Comitê de Ética da Universidade
Federal de Santa Catarina projeto aprovado sob número 008/07 em 26/03/2007).

A entrevista e as filmagens dos professores no Mix 30 foram realizadas nos locais das aulas. Para a filmagem, a câmara foi fixada em um tripé e posicionada a $1,5 \mathrm{~m}$ de altura e a 5 metros de distância do professor, de modo que as imagens capturadas fossem no plano do movimento (plano sagital). As medidas de massa, estatura e impacto foram coletadas no Laboratório de Biomecânica da Universidade Federal de Santa Catarina. As entrevistas, as filmagens e as medidas no laboratório foram efetuadas com agendamento prévio individual. Para as medidas de massa e estatura, utilizou-se o protocolo de Petroski (2007). Para as medidas das magnitudes de impacto foi seguido o protocolo de Santos (2003), sendo que o acelerômetro inicialmente foi fixado no joelho (sobre a articulação tibio-femural) e posteriormente no tornozelo (logo acima do maléolo lateral da tíbia) da perna de aterrissagem. A fixação do acelerômetro e dos cabos foi efetuada com fita elástica para permitir o livre movimento do professor para a realização do Jump Kick, pressionada de forma que não houvesse oscilação entre o acelerômetro e o local fixado. Foram realizados 10 saltos para as medidas em cada segmento corporal, com intervalo de 30 segundos entre eles, sem a utilização de música.

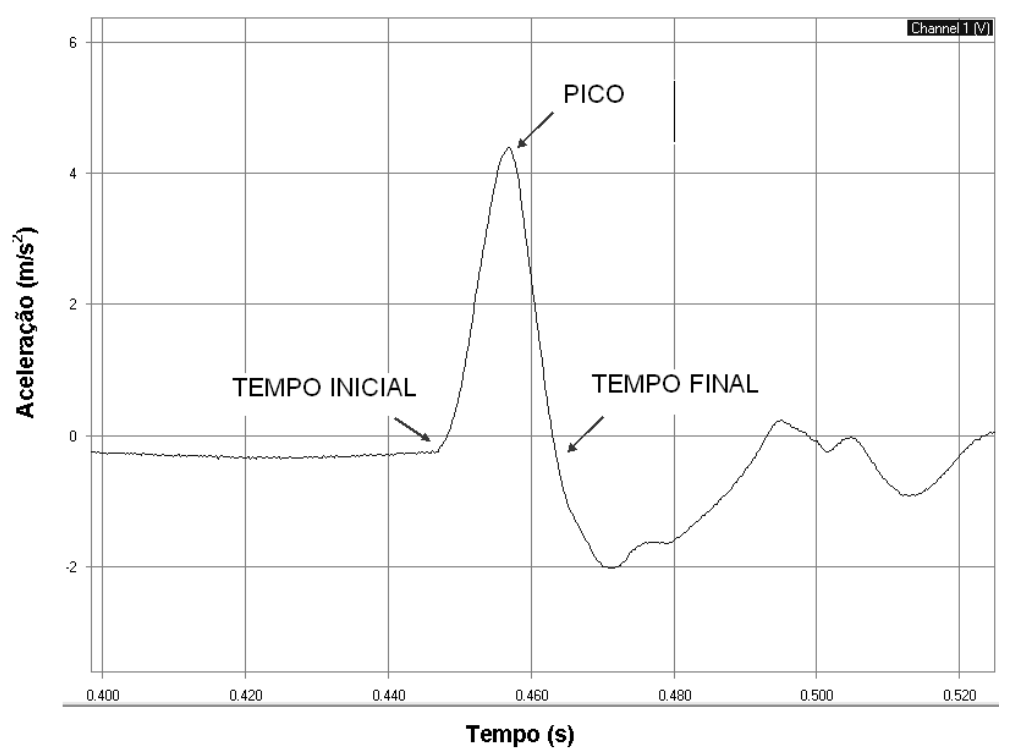

Figura 1. Exemplo do critério adotado para obter o valor do pico e do tempo do pico em cada eixo a partir do programa AqDados 7.02. 
Para o tratamento dos dados, utilizou-se o pico de impacto (Figura 1), sendo que os sinais advindos do acelerômetro $\left(\mathrm{m} / \mathrm{s}^{2}\right)$ nas três direções $(x, y \mathrm{e} z)$ foram tabulados em uma planilha do programa Microsoft Excel ${ }^{\circledR}$, corrigidos pelos fatores de pré-amplificação específicos para cada eixo, definidos de acordo com a especificidade do salto de cada professor e divididos por $9,81 \mathrm{~m} / \mathrm{s}^{2}$ para serem transformados em unidades de aceleração da gravidade $(g)$.

Para a análise estatística foi utilizado o teste Shapiro Wilk para verificar a normalidade dos dados. Para identificar as magnitudes e os tempos de impacto no tornozelo e no joelho e o número de impactos (saltos) nas aulas, foi utilizada estatística descritiva (média, desvio padrão e coeficiente de variação); para a comparação dos dados entre as articulações, entre os eixos e entre os sujeitos, utilizou-se análise de variância multi-fatorial; para verificar a relação entre a massa corporal, a estatura e o tempo de prática com as magnitudes de impacto, utilizou-se a correlação linear de Pearson. Para todos os testes foi adotado $p \leq 0,05$.

\section{Resultados}

A partir das filmagens dos professores ministrando as aulas no Mix 30 , observou-se que o referido mix é composto por 1420 saltos, ou seja, em uma aula todos os sujeitos executaram 1420 saltos, sendo que 312 saltos foram realizados com a perna esquerda e direita de maneira alternada, e $796 \mathrm{com}$ ambas as pernas. Assim, levando em consideração o número de aulas ministradas pelos professores, eles realizam em média $4.792 \pm 2.138$ saltos por semana; $17.927 \pm 10.395$ por mês $e$ $127.800 \pm 93.775$ no trimestre.

Quanto as magnitudes e os tempos de impacto no tornozelo e no joelho advindos das aterrissagens do salto Jump Kick, os dados são apresentados na Tabela 1 e 2, respectivamente.

Tabela 1. Magnitudes e tempos de impacto registrados no joelho nos eixos antero-posterior $(x)$, vertical (y) e latero-lateral ( $z$ ) na execução da técnica do Jump Kick.

\begin{tabular}{|c|c|c|c|c|}
\hline \multirow{4}{*}{$\begin{array}{l}\text { Magnitude } \\
(g)\end{array}$} & & $x$ & $y$ & $z$ \\
\hline & Média & 13,04 & 8,98 & 6,61 \\
\hline & DP & 6,51 & 3,93 & 2,18 \\
\hline & $\mathrm{CV}$ & 49,91 & 43,75 & 33,12 \\
\hline \multirow{3}{*}{ Tempo (s) } & Média & 0,028 & 0,022 & 0,021 \\
\hline & DP & 0,007 & 0,005 & 0,006 \\
\hline & CV & 25,23 & 25,20 & 27,53 \\
\hline
\end{tabular}

Tabela 2. Magnitudes e tempos de impacto registrados no tornozelo nos eixos anteroposterior $(x)$, vertical $(y)$ e latero-lateral $(z)$ na execução da técnica do Jump Kick.

\begin{tabular}{|c|c|c|c|c|}
\hline \multirow{4}{*}{$\begin{array}{l}\text { Magnitude } \\
(g)\end{array}$} & & $x$ & $y$ & $z$ \\
\hline & Média & 18,97 & 11,18 & 9,71 \\
\hline & DP & 9,08 & 2,77 & 5,61 \\
\hline & $\mathrm{CV}$ & 47,86 & 24,79 & 57,75 \\
\hline \multirow{3}{*}{ Tempo (s) } & Média & 0,021 & 0,019 & 0,019 \\
\hline & DP & 0,005 & 0,004 & 0,002 \\
\hline & $\mathrm{CV}$ & 24,50 & 21,10 & 12,84 \\
\hline
\end{tabular}

A partir dos resultados da Tabela 1 e 2, observa-se que os maiores valores de impacto ocorreram na articulação do tornozelo, nos três eixos. Verifica-se também uma alta variabilidade dos dados $(C V>30 \%)$ nas duas articulações em todos os eixos.

No que concerne às comparações das magnitudes dos impactos entre os sujeitos, entre os eixos e entre as articulações, os resultados estão apresentados na Tabela 3.

Tabela 3. Comparação das magnitudes de impacto entre sujeitos, eixos e articulações de joelho e tornozelo na execução do Jump Kick.

\begin{tabular}{|c|c|c|c|c|}
\hline & Sujeitos & $x$ & $y$ & $z$ \\
\hline \multirow{8}{*}{ Joelho } & 1 & 6,8 & 11,8 & 11,7 \\
\hline & 2 & 11,9 & 9,6 & 6,5 \\
\hline & 3 & 22,4 * & 12,5 & 9,6 \\
\hline & 4 & 12,4 & 14,1 & 9,3 \\
\hline & 5 & 9,2 & 13,3 & 13,8 \\
\hline & 6 & 14,7 & 5,2 & 5,5 \\
\hline & 7 & 15,8 & 13,8 & 15,4 \\
\hline & 8 & 6,8 & 10,6 & 6,1 \\
\hline \multirow{8}{*}{ Tornozelo } & 1 & 10,6 * & 8,4 & 11,5 \\
\hline & 2 & $30,0 * \Psi$ & 27,6 * $\Psi$ & $17,4^{\Psi \xi}$ \\
\hline & 3 & 23,2 & 12,6 & 12,4 \\
\hline & 4 & $31,4 * \Psi$ & 14,5 & $21,6^{\Psi}$ \\
\hline & 5 & 15,1 & 8,5 & 6,0 * \\
\hline & 6 & 16,2 & 9,5 & 6,4 * \\
\hline & 7 & 31,4 * & 14,5 & 21,6 \\
\hline & 8 & 9,9 * & 10,5 & 6,2 * \\
\hline
\end{tabular}

Analisando a Tabela 3, encontrou-se diferença entre os sujeitos, apenas do indivíduo três $(22,4$ $g$ ) em relação ao restante do grupo no eixo $x$ da articulação do joelho. No eixo $x$ do tornozelo, os 
professores de número um $(10,6 \mathrm{~g})$ e oito $(9,9 \mathrm{~g})$ apresentaram valores significativamente menores do restante do grupo. No eixo $y$, apenas o professor de número dois apresentou valor significativa maior $(27,6 \mathrm{~g})$ do restante do grupo. No eixo $z$, os professores cinco, seis e oito apresentaram valores significativamente menores (6,0 g, 6,4 g e 6,2 g, respectivamente) do restante do grupo. Quanto à diferença entre as articulações corporais, o tornozelo obteve valores significativamente maiores para os professores de número dois $(30,0 \mathrm{~g})$ e quatro $(31,4 \mathrm{~g})$ no eixo $x$, e para o professor dois no eixo $y(27,6 g)$ e eixo $z(17,4 \mathrm{~g})$ e para o professor quatro no eixo $z$ $(21,6 \mathrm{~g})$. Considerando a diferença entre os eixos, só o professor dois teve as medidas no tornozelo $(17,4 \mathrm{~g})$ eixo $z$, significativamente menor que os demais.

No que concerne às correlações entre as variáveis magnitudes de impacto obtidas na aterrissagem do Jump Kick com massa corporal, estatura e experiência em ministrar aulas de Body Combat, não houve nenhuma correlação estatisticamente significante.

\section{Discussão}

Com relação ao número médio de saltos que os professores realizam durante a prática de Body Combat, considerando apenas o Mix 30, pode-se afirmar que este número é extremamente alto e preocupante, pois de acordo Radin et al (1972), as sobrecargas advindas de saltos ao corpo humano é uma possível causa de quebra de cartilagens e degeneração de articulações. Outros pesquisadores investigaram as relações entre a repetição de movimentos impactantes e o acometimento de lesões em diferentes modalidades, como no basquetebol (PIUCCO et al., 2007), voleibol (SANTOS et al., 2007a), handebol (SANTOS et al., 2007b) e corrida (HAMILL et al., 1995). Porém, mesmo que não existam padronizações e evidências empíricas, os estudos apontam que os impactos repetitivos sofridos por atletas são um dos principais responsáveis pelo acometimento de lesões (VOLOSHIN, 2004; LAFORTUNE et al. 1995; DERRICK et al., 1998). Em algumas medidas subjetivas, obtidas de acordo com o tempo de exposição, foi claramente demonstrado que as condições de impactos e vibrações transitórias e com grande amplitude de maneira resultaram em dor e desconforto remanescente (MORRISON et al. 1999).
Alguns estudos relacionam o aumento das magnitudes dos impactos com o surgimento da fadiga (VERBITSKY et al. 1998; MIZRAHI et al. 2000) e que com a aplicação de cargas excessivas, as sínteses celulares dentro da cartilagem articular podem diminuir, e a degradação aumentar, causando as modificações de deterioração pelo uso comum que acompanham a prática prolongada de exercício (VAZAN, 1983).

Quanto às magnitudes e os tempos dos impactos nas articulações do joelho e tornozelo (Tabelas 1 e 2), de acordo com o critério de Gomes (1990), verifica-se uma alta variabilidade dos dados. Este fato pode ser justificado pela coleta ter sido realizada em laboratório, bem como pela dificuldade dos professores de manterem um padrão tanto do salto quanto da aterrissagem da técnica.

Ainda, conforme os resultados das Tabelas 1 e 2, verifica-se que os valores de impacto no joelho são menores do que no tornozelo durante a aterrissagem. As forças de impacto durante aterrissagens são absorvidas pelo corpo por vários elementos, principalmente pela cartilagem articular, visto que ela distribui as cargas sobre a sua superfície e reduz o estresse de contato ósseo pela metade (HETTINGA, 1985; WIECZOREK et al., 1997). No joelho, a cartilagem articular é mais espessa do que no tornozelo, pois, apesar das cargas nessas articulações serem menores, a área de distribuição dessas cargas também é menor, impondo mais estresse sobre a cartilagem (WALLACE et al., 1985).

As magnitudes de impacto geradas durante as aterrissagens no Body Combat foram menores do que as magnitudes de impacto encontradas durante as aterrissagens de saltos na bandeja do basquetebol $(53,23 \mathrm{~g}$ no eixo vertical do tornozelo) e na cortada do voleibol $(50,78 \mathrm{~g}$ no eixo vertical do tornozelo) (PIUCCO; SANTOS, 2009). As baixas magnitudes de impactos encontradas neste estudo podem ter ocorrido devido a aterrissagem realizada apenas com uma das pernas, ao contrário das aterrissagens de saltos de bloqueio no voleibol e bandeja no basquetebol. Outro fator que justifica as baixas magnitudes de impacto nas aterrissagens do Jump Kick é que o professor não efetua uma corrida de aproximação ou de impulsão para realizar os saltos, diferentemente das outras 
modalidades investigadas (basquetebol, voleibol, handebol).

Os valores de tempo de duração dos impactos aqui obtidos foram semelhantes aos encontrados em aterrissagens no voleibol (SANTOS, et al. 2007a), no basquetebol (PIUCCO, et al. 2007) e na corrida (LAFORTUNE et al., 1995), sendo estes valores de tempo de duração dos impactos nos saltos foram $<10^{-2} \mathrm{~s}$.

A relação entre magnitude e tempo de impacto é de suma importância, pois o tempo de exposição a qualquer tipo de vibração é que determinará os riscos para o acometimento de lesão (ISO 2631, 1985). As normas existentes que determinam limites para a exposição às vibrações ao corpo humano, como a norma ISO 2631, estabelecem curvas que vão de $1 \mathrm{~min}$ (um minuto) até 24 h (vinte e quatro horas), não sendo aplicadas as vibrações transitórias de curta duração, como é o caso dos impactos nos esportes e na prática do Body System. O único critério que se pode tomar como referência (entendendo a duração do pulso como o tempo total de impacto) para ser utilizado neste estudo, é o citado por Macaulay (1987). De acordo com esse critério, pode-se afirmar que os valores de tempo obtidos neste estudo, tanto no joelho quanto no tornozelo, podem ser considerados de curta duração, e os valores dos impactos nestas articulações são baixos, estando, portanto, dentro dos limites considerados seguros conforme o critério supracitado. Por outro lado, apesar do critério de Macaulay ser o único que pode ser usado para referenciar as vibrações transitórias, características dos impactos esportivos, esse critério não cita o número de impactos, o período de exposição, nem o intervalo entre as repetições dos mesmos que sejam mínimos para que não haja lesões. Sem o controle destas variáveis não se pode fazer qualquer inferência sobre a capacidade de auto-reparo dos biomateriais frente às características das cargas e ao processo de fadiga, advindo nas aterrissagens do Jump Kick. Pois, quanto menores forem as durações dos impactos, maiores são as magnitudes que o corpo pode sustentar e somente os impactos não são suficientes para o acometimento de danos, mas $\operatorname{sim}$ a repetição destes de maneira continua no organismo (RADIN et al., 1998).

Ainda, alguns estudos como os de Verbitsky et al. (1998) e Mizrahi et al. (2000) relatam que o aumento nas magnitudes dos impactos aumenta com o surgimento da fadiga. O aumento da fadiga, associado ao grande número de aterrissagens realizadas nas aulas de Body Combat, podem ser responsáveis por possíveis efeitos deletérios ao organismo dos professores de Body Combat ao longo do tempo de prática. Vasan (1983) explica que com a aplicação de cargas excessivas, as sínteses celulares dentro da cartilagem articular podem diminuir, e a degradação aumentar, causando as modificações de deterioração pelo uso comum que acompanham a prática prolongada de exercício.

Quanto à comparação das magnitudes de impacto entre os sujeitos, os eixos e as articulações (Tabela 3), as diferenças encontradas podem ser explicadas pela forma de execução da técnica de amortecimento da queda deste salto. A técnica adequada de aterrissagem tem como função principal suavizar o impacto gerado durante o salto. Deste modo, a forma de amortecer a aterrissagem do salto do Jump Kick é um dos fatores que contribuem para o aumento ou redução das cargas que contribuirão para a aquisição de lesões em longo prazo. Zhang et al. (2005) e Self e Paine (2001) concordam que o melhor tipo de aterrissagem de saltos é iniciar o contato a partir da ponta dos pés (artelhos), e transferir as cargas de impacto até o contato dos calcanhares contra o solo.

O resultado da correlação permite afirmar que, para este grupo de professores, a massa corporal, a estatura e a experiência em ministrar aulas de Body Combat não parecem influenciar nas magnitudes de impacto obtidas na aterrissagem do Jump Kick. Resultados diferentes foram obtidos por Piucco e Santos (2008) que encontraram correlação positiva $(r=0,85)$ entre a massa corporal e os impactos gerados no tornozelo (eixo vertical) durante aterrissagens da cortada no voleibol, bem como Piucco e Santos (2009) que encontraram correlação positiva $(r=0,73)$ entre o $\% G$ com as magnitudes dos impactos no tornozelo.

Visto que as forças de impacto dependem diretamente da massa dos corpos envolvidos na colisão, bem como da velocidade de contato entre os corpos num dado intervalo de tempo, como o piso é estático durante a aterrissagem, somente a massa do sujeito é considerada, influenciando nas magnitudes dos impactos (NUSSENZVEIG, 1996). A não correlação das 
variáveis neste estudo pode ter ocorrido pelas baixas magnitudes de impacto geradas durante as aterrissagens no Body Combat, quando comparadas com os estudos citados.

Para as magnitudes dos impactos no joelho não foram encontrados estudos que obtiveram correlação com a massa corporal durante aterrissagens. Uma possível explicação para a não correlação entre essas variáveis é a realização da técnica de amortecimento durante a aterrissagem, caracterizada pela flexão do joelho no sentido ântero-posterior, que contribui para amenizar os efeitos dos impactos, independente da massa corporal (HAMILL; KNUTZEN, 1999; LAFORTUNE et al, 1996).

Por fim, mesmo que a média das magnitudes e dos tempos dos impactos dos professores, segundo o critério de Macaulay (1987), sejam valores considerados como não causadores de lesões, vale ressaltar que o número de saltos que os professores realizam por dia, semana e no trimestre, poderão causar efeitos deletérios ao organismo de maneira crônica, principalmente danos articulares.

\section{Conclusões}

Tendo em vista as condições em que foi realizado o estudo e com base no referencial teórico, foi possível concluir que:

a) a repetição de saltos durante a jornada diária, semanal e conseqüentemente, mensal de trabalho é extremamente alta;

b) os impactos apresentados na aterrissagem da técnica Jump Kick são considerados eventos de baixa magnitude e de curta duração, classificados como não causadores de lesões;

c) as diferenças nas magnitudes dos impactos encontradas entre os professores, bem como a heterogeneidade dos dados, confirmam o pouco domínio do movimento;

d) a massa corporal, a estatura e o tempo de prática não interferiram nas magnitudes dos impactos dos professores realizando a técnica Jump Kick.

Considerando as magnitudes, os tempos de impacto nos membros inferiores bem como o número de saltos que os professores de Body Combat realizam no seu cotidiano de trabalho, medidas preventivas devem ser tomadas a fim de evitar a ocorrência de lesões causadas pelas sobrecargas nos membros inferiores.

\section{Referências}

AMADIO, A. C. et al. Introdução à Biomecânica para Análise do Movimento Humano: Descrição e Aplicação dos Métodos de Medição. Revista Brasileira de Fisioterapia, v. 3, n. 2, p. 4154,1999 .

BRAUNER, V. L. P. Novos Sistemas de Aulas de Ginástica, Procedimentos Didáticos na Formação dos Professores. Revista Brasileira de Medicina do Esporte, v. 28, n. 2, p. 211-219, 2007.

COELHO, G. F. M.; TRIVIA, R. C.; PIUCCO, T. Relação entre tempos e magnitudes de impactos nas aterrissagens de bandejas e rebotes no basquetebol. In: CONGRESSO BRASILEIRO DE BIOMECÂNICA, São Pedro, 7, 2007. Anais... São Pedro: USP, 2007. p. 793-798.

COVENTRY, E. et al. The effect of lower extremity fatigue on shock attenuation during single-leg landing. Clinical Biomechanics, v. 21, p. 1090-1097, 2006.

DERRICK, T. R.; HAMIL, J.; CALDWEL, G. E. Energy Absorption of impacts during running at various stride lengths. Medicine and Science in Sports Exercises, v. 30, n.1, p. 128-135, 1998.

DUFEK, J. S.; ZHANG, S. Landing models for volleyball players: a longitudinal evaluation. Journal of Sports Science and Medicine and Physical Fitness, v. 36, n. 1, p. 35-42, 1996.

FANTINI, C.; MENZEL, H. J. Análise de impactos em aterrissagens após saltos máximos em diferentes grupos de atletas e não-atletas. In: CONGRESSO BRASILEIRO DE BIOMECÂNICA, 9., 2001, Gramado. Anais... Gramado: UFRGS, 2001, v. 2, p. 89-93.

GOMES, F. P. Curso de estatística experimental. 13. ed. Piracicaba: Nobel AS. 1990

HAMILL, J.; KNUTZEN, K. M. Bases Biomecânicas do Movimento Humano. São Paulo: Manole, 1999.

HAMILL, J.; DERRICK, T. R; HOLT, K. G. Shock attenuation and stride frequency during running. Human Movement Science, v. 14, p. 45-60, 1995.

HETTINGA, D. L. Inflammatory response of synovial joint structures. In: GOULD, J. A; DAVIES, G. J. (Eds). Orthopedic and Sports Physical Therapy, St. Louis: C.V. Mosby, 1985, p. 87-117.

INTERNATIONAL STANDARD. Evaluation of human exposure to whole-body vibration. Part 
1: General requirements. ISO 2631, p. 1-17, 1985.

LAFORTUNE, M. A.; HENNIG, E. M.; LAKE, M. J. Dominant role of interface over knee angle for cushioning impact loading and regulating initial leg stiffness. Journal of Biomechanics, v. 29, n. 12, p. 1523-1529, 1996.

LAFORTUNE, M. A.; HENNING, E.; VALIANT, G. $A$, Tibial shock measured with bone and skin mounted transducers. Journal of Biomechanics, v. 28, n. 8, p. 989-93, 1995

MACAULAY, M. Introduction to impact engineering. London: Chapman and Hall, 1987.

MAHAR, A. T.; DERRICK, T. R.; HAMILL, J.; CALDWELL, G. E. Impact shock and attenuation during in-line skating. Medicine \& Science in Sports \& Exercises, v. 29, p. 1069-1075, 1997.

MERCER, J. A.; DEVITA, P.; DERRICK, T. R.; BATES, B. T. Individual effects of stride length and frequency on shock attenuation during running. Medicine \& Science in Sports \& Exercise, v. 35, n. 2, p.307-313, 2003b.

MILLS, L. Body Combat. Instructor Manual. Les Mills International, Auckland. 2002.

MIZRAHI, J.; VERBITSKY, O.; ISAKOV, E. Shock accelerations and attenuation in downhill and level running. Clinical Biomechanics, v. 15, p. 15-20, 2000.

MORRISON, J. B.; ROBINSON, D. G.; NICOL, J. et al. A biomechanical approach to evaluating the health effects of repeated mechanical shocks. Proceedings Human Factors and Medicine Panel, v. 20, Cedex, France, 1999, pp. 24.1-24.8

NIGG, B. M. Impact forces in running. Current Opinion in Orthopedics, v. 8, p. 43-47, 1997.

NIGG, B. M.; WAKELING, J. M. Impact forces and muscle tuning: a new paradigm. Exercise and Sport Science Reviews, v. 29, p. 37-41, 2001.

NUSSENZVEIG, H. M. Curso de física básica 1 - mecânica. $3^{a}$ ed. São Paulo: Edgard Blucher, 1996.

PANDA, M. D. J. Estudo dinâmico dos principais padrões de passos do step training. 2001. Dissertação (Mestrado em Ciência do Movimento Humano) Centro de Educação Física, Fisioterapia e Desportos, Universidade do Estado de Santa Catarina, Florianópolis, 2001.

PETROSKI, E. L. (organizador). Antropometria: técnicas e padronizações. 3 ed. Blumenau: Nova Letra, 2007.
PIUCCO, T; SOUZA, P.D.;PACHECO, A.G. et al. Magnitude dos impactos durante as aterrissagens no basquete associado com lesões nos membros inferiores. In: CONGRESSO BRASILEIRO DE BIOMECÂNICA, 12., 2007, Rio Claro. Anais... Rio Claro, 2007. p. 781-786.

PIUCCO, T.; SANTOS, S. G. Características de impacto em diferentes regiões corporais durante a queda na técnica o-soto-gari. Lecturas Educación Física y Deportes, v. 13, n.125, p.12, 2008.

PIUCCO, T.; SANTOS, S. G. Relação entre percentual de gordura corporal, desempenho no salto vertical e impacto nos membros inferiores em atletas de voleibol. Fitness and Performance Journal, v.8, n.1, p.9-15, 2009.

RADIN, E. L.; PAUL, I. L.; ROSE, R. M. Role of mechanics factors in pathogenesis of primary osteoarthritis. Lancet, v.4, n.1, p.519-522, 1972.

SACCO, I. et al. Influência de implemento s para o tornozelo nas respostas biomecânicas do salto e aterrissagem no basquete. Revista Brasileira de Medicina do Esporte. v. 10. n. 6, 2004.

SANTOS, S. G. Estudo das características de impacto e percepção humana de conforto na prática de ukemis em diferentes tatamis. 2003. $177 \mathrm{f}$. Tese (Doutorado em Engenharia de Produção e Sistemas) Universidade Federal de Santa Catarina, Florianópolis, 2003.

SANTOS, S. G.; DETANICO, D.; GRAUP, S.; REIS, D. C. Relação entre alterações posturais, prevalência de lesões e magnitude de impacto nos membros inferiores em atletas de handebol. Fitness and Performance Journal, v. 6, n. 6, p. 388-393, 2007a.

SANTOS, S. G.; PIUCCO, T.; REIS, D. C. Fatores que interferem nas lesões de atletas amadores de voleibol. Revista Brasileira de

Cineantropometria e Desempenho Humano, v. 9, n. 2, p. 189-195, 2007b.

SELF, B.P.; PAINE, D. Ankle biomechanics during four landing techniques. Medicine and Science in Sports Exercises, v. 33, n. 8, p. 1338-1344, 2001.

VASAN, N. Effects of physical stress on the synthesis and degradation of cartilage matrix. Connective Tissue Research, v.12, p. 49-58, 1983.

VERBITSKY, O.; MIZRAHI, J.; VOLOSHIN, A.; TREIGER, J.; IZAKOV, E. Shock transmission and fatigue in human running. Journal of Applied Biomechanical, v. 14, p. 300-311, 1998. 
VOLOSHIN, A. S. Propagação do Impacto e seus Efeitos sobre o Corpo Humano. In: ZATSIORSKY V. Biomecânica no Esporte: Performance e Prevenção da Lesão. Rio de Janeiro: Guanabara Koogan; 2004.

WALLACE, L. A.; MANGINE, R. E.; MALONE, T. The knee. In: GOULD, J. A.; DAVIES, G. J. (Eds). Orthopedics and Sports Physical Therapy. St Louis: CV Mosby, 1985. cap. 16. p 359-363.

WIECZOREK, A. S; DUARTE, M.; AMADIO, A. C. Estudo da força reação do solo no movimento básico de step. Revista Paulista de Educação Física, v. 11, n. 2, p. 103- 15, 1997.

ZHANG, S. et al. Effects of various midsole densities of basketball shoes on impact attenuation during landing activities. Journal of Applied Biomechanics, v. 21, n. 1, p. 3-17, 2005.

O trabalho apresentado foi derivado da dissertação de mestrado, apresentada ao Programa de Pós-Graduação em Educação Física, Universidade Federal de Santa Catarina, para obtenção do título de Mestre em Educação Física (Área da Cineantropometria e Desempenho Humano).

\section{Endereço:}

Saray Giovana dos Santos

Rua Maria Eduarda, 506, Pantanal,

Florianópolis, SC, Brasil

88050-250

Telefone: (48) 9919-3915 Fax: (48) 3721-8530

e-mail: senseisaray@hotmail.com

Recebido em: 28 de abril de 2009.

Aceito em: 23 de dezembro de 2009.

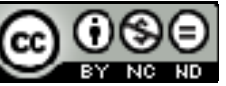

Motriz. Revista de Educação Física. UNESP, Rio Claro, SP, Brasil - elSSN: 1980-6574 - está licenciada sob Licença Creative Commons 